\title{
INTERFUEL: FAST - FY 2020 Federal Fleet Dataset
}

\section{March 2021}

Ron Stewart

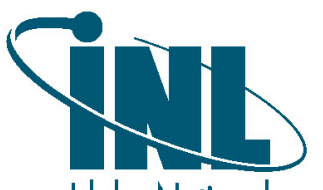

Idaho National

Laboratory 


\section{DISCLAIMER}

This information was prepared as an account of work sponsored by an agency of the U.S. Government. Neither the U.S. Government nor any agency thereof, nor any of their employees, makes any warranty, expressed or implied, or assumes any legal liability or responsibility for the accuracy, completeness, or usefulness, of any information, apparatus, product, or process disclosed, or represents that its use would not infringe privately owned rights. References herein to any specific commercial product, process, or service by trade name, trade mark, manufacturer, or otherwise, does not necessarily constitute or imply its endorsement, recommendation, or favoring by the U.S. Government or any agency thereof. The views and opinions of authors expressed herein do not necessarily state or reflect those of the U.S. Government or any agency thereof. 


\title{
INTERFUEL: FAST - FY 2020 Federal Fleet Dataset
}

\author{
Ron Stewart
}

March 2021

\section{Idaho National Laboratory \\ Idaho Falls, Idaho 83415}

http://www.inl.gov

\author{
Prepared for the \\ U.S. Department of Energy \\ Under DOE Idaho Operations Office \\ Contract DE-AC07-05ID14517, DE-AC07-05ID14517
}


FAST: FY 2020 Federal Fleet Dataset INTERFUEL •March 2021 


\section{FY 2020 Federal Fleet Dataset: Overview}

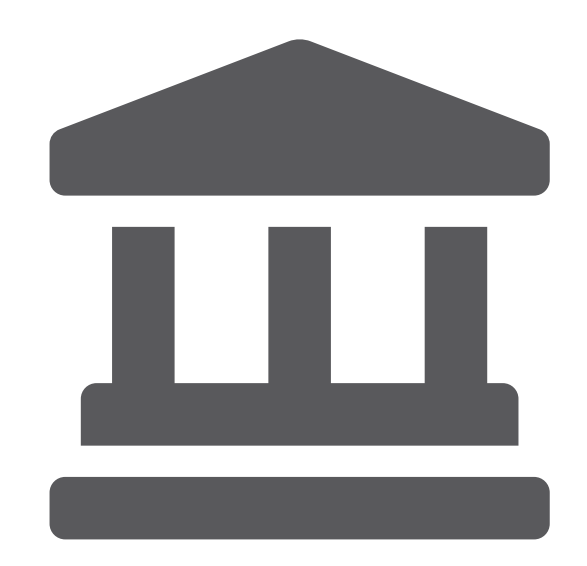

49 organizations

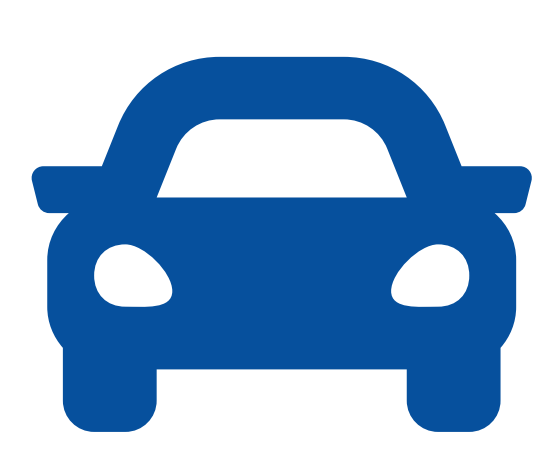

704K vehicles

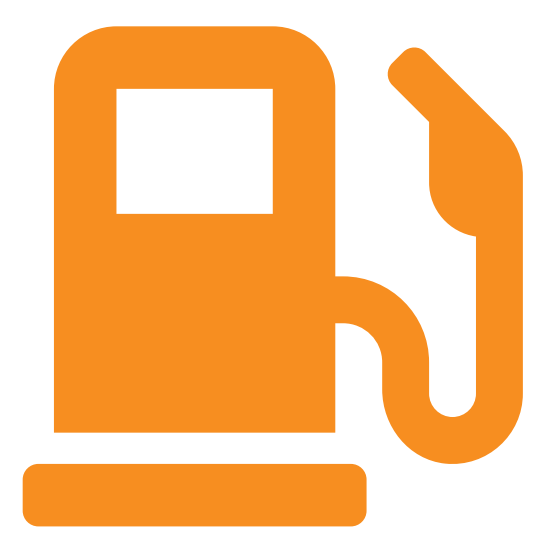

$1.1 \mathrm{M}$ fuel records 


\section{FY 2020 Federal Fleet Dataset: Overview}

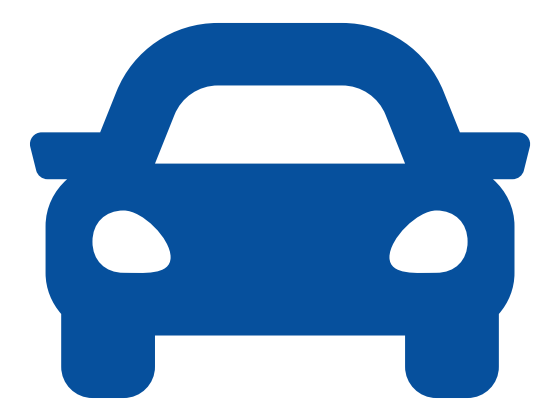

Inventory $658 \mathrm{~K}$ $+1.9 \%$

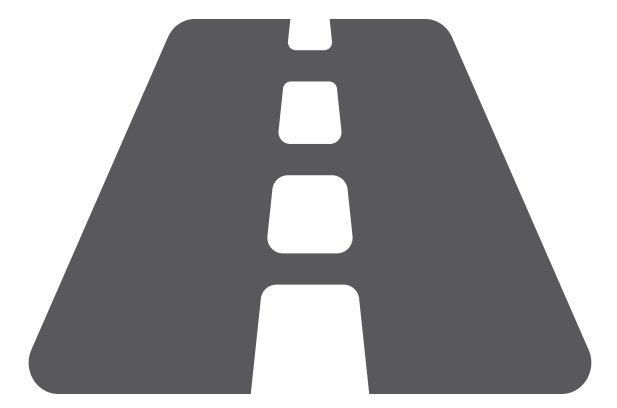

Miles

4.16B

$-7.1 \%$

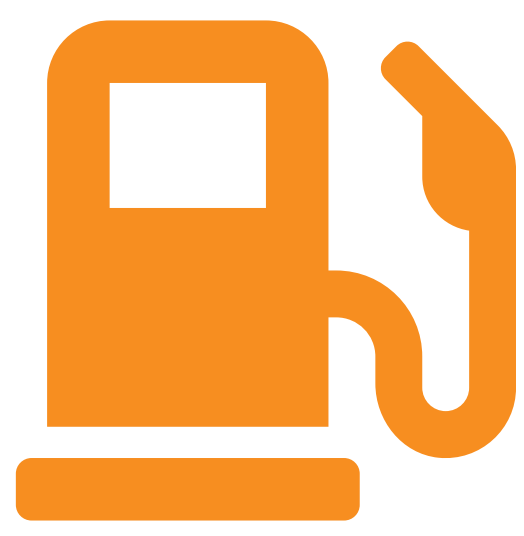

Fuel 372M GGE

$-3.8 \%$

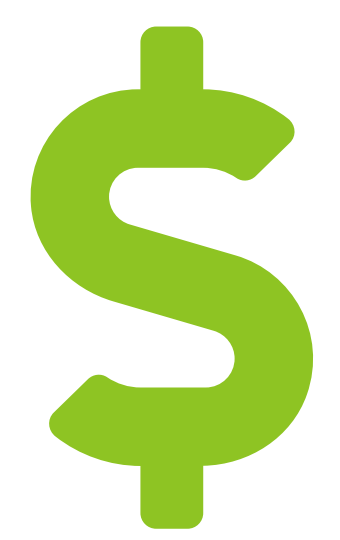

Cost $\$ 3.55 \mathrm{~B}$

$-3.9 \%$ 


\section{FY Federal 2020 Fleet Dataset: Overview}

Agency Submissions

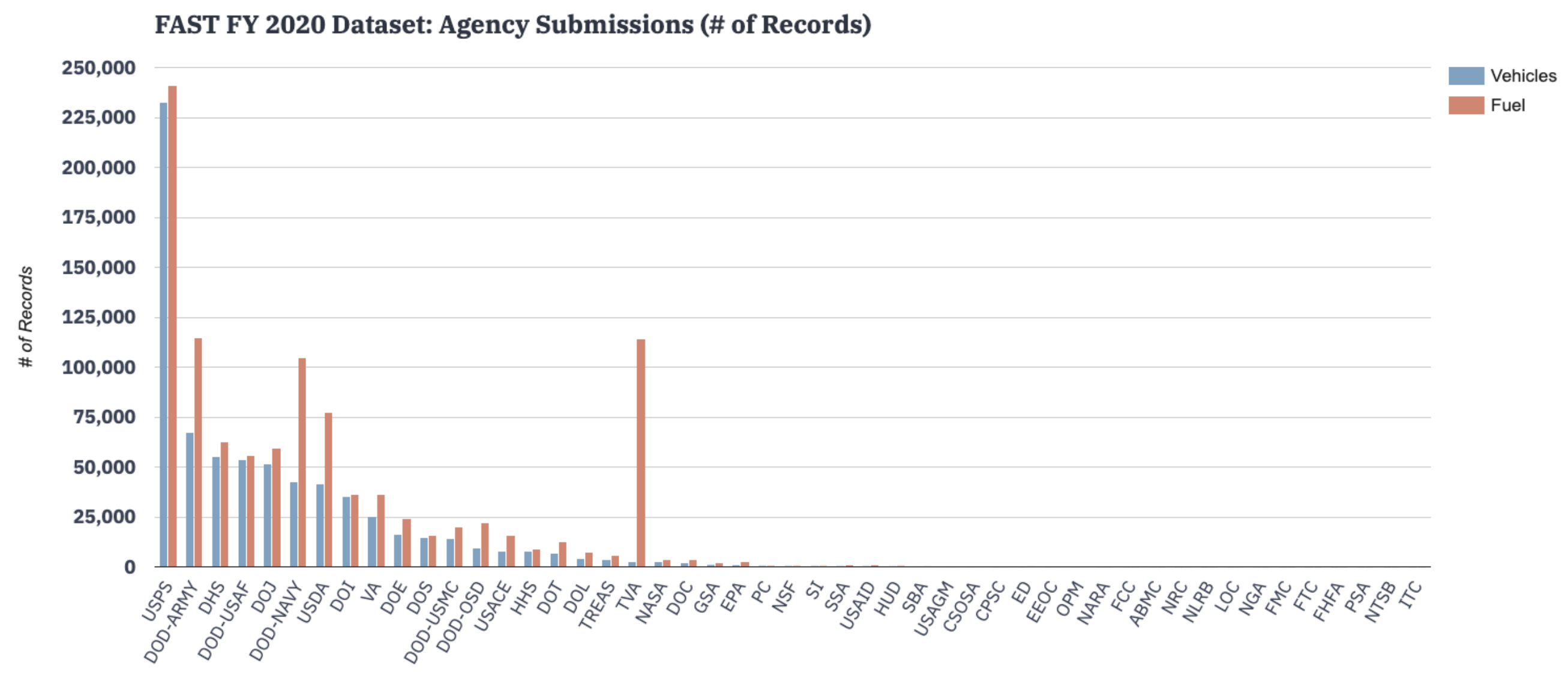




\section{FY 2020 Federal Fleet Dataset: Overview}

Agency Submissions

FAST FY 2020 Dataset: Agency Submissions (\# of Records)

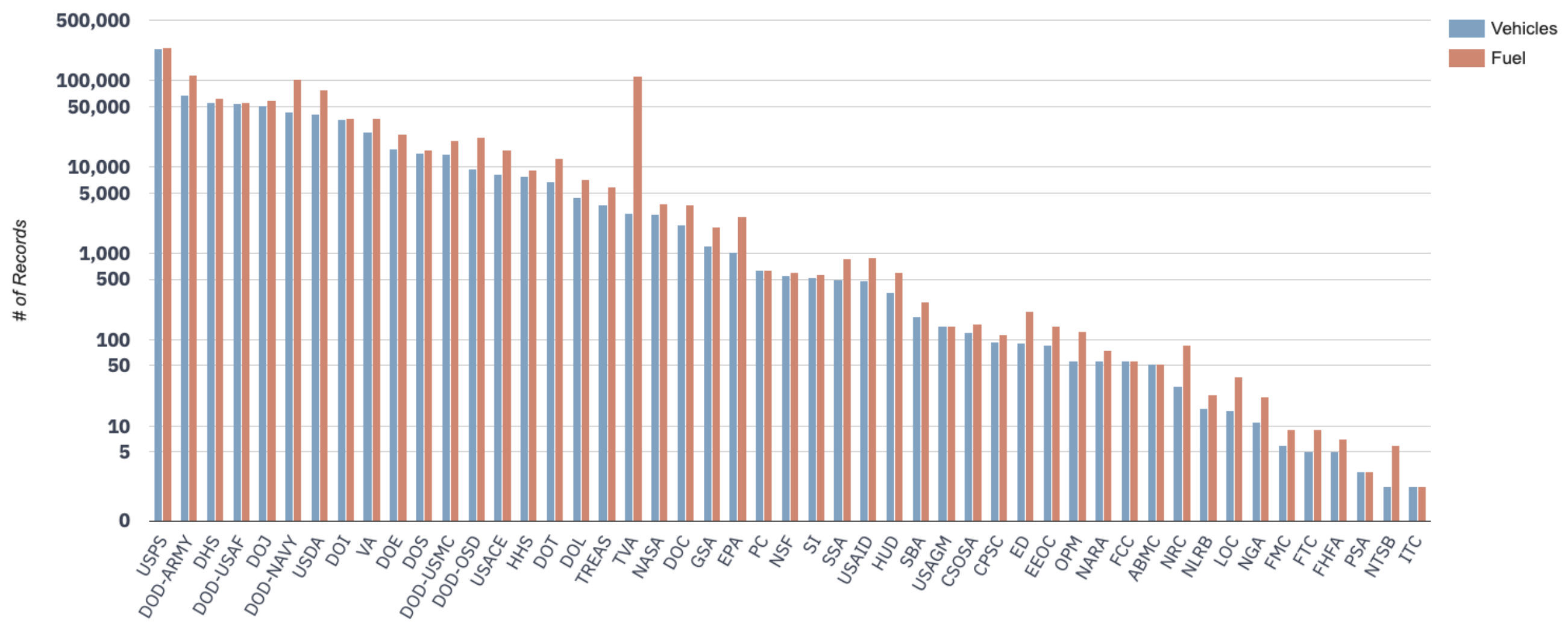




\section{FY 2020 Federal Fleet Dataset: 5-Year Trends}

Vehicle Inventory

FAST FY 2020 Dataset: Vehicle Inventory by Ownership

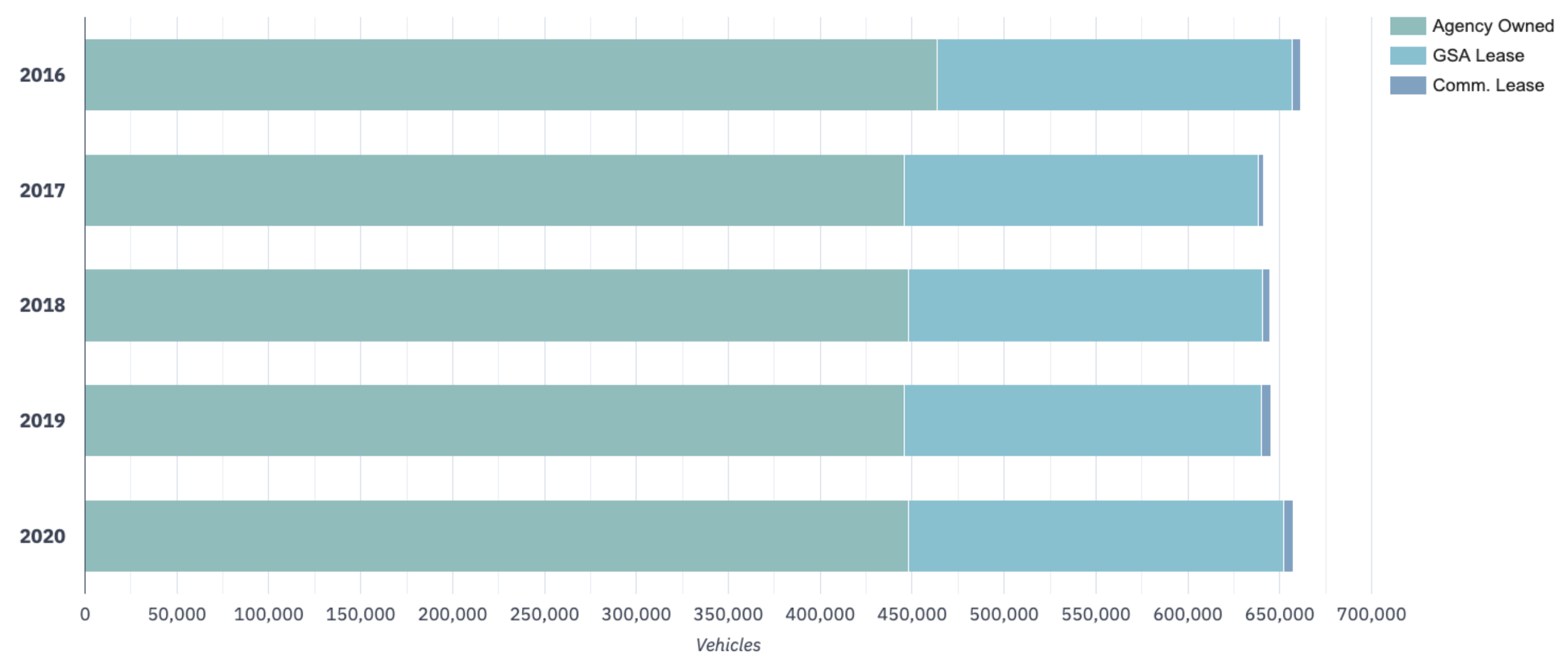




\section{FY 2020 Federal Fleet Dataset: 5-Year Trends}

Vehicle Inventory

FAST FY 2020 Dataset: Vehicle Inventory by Ownership

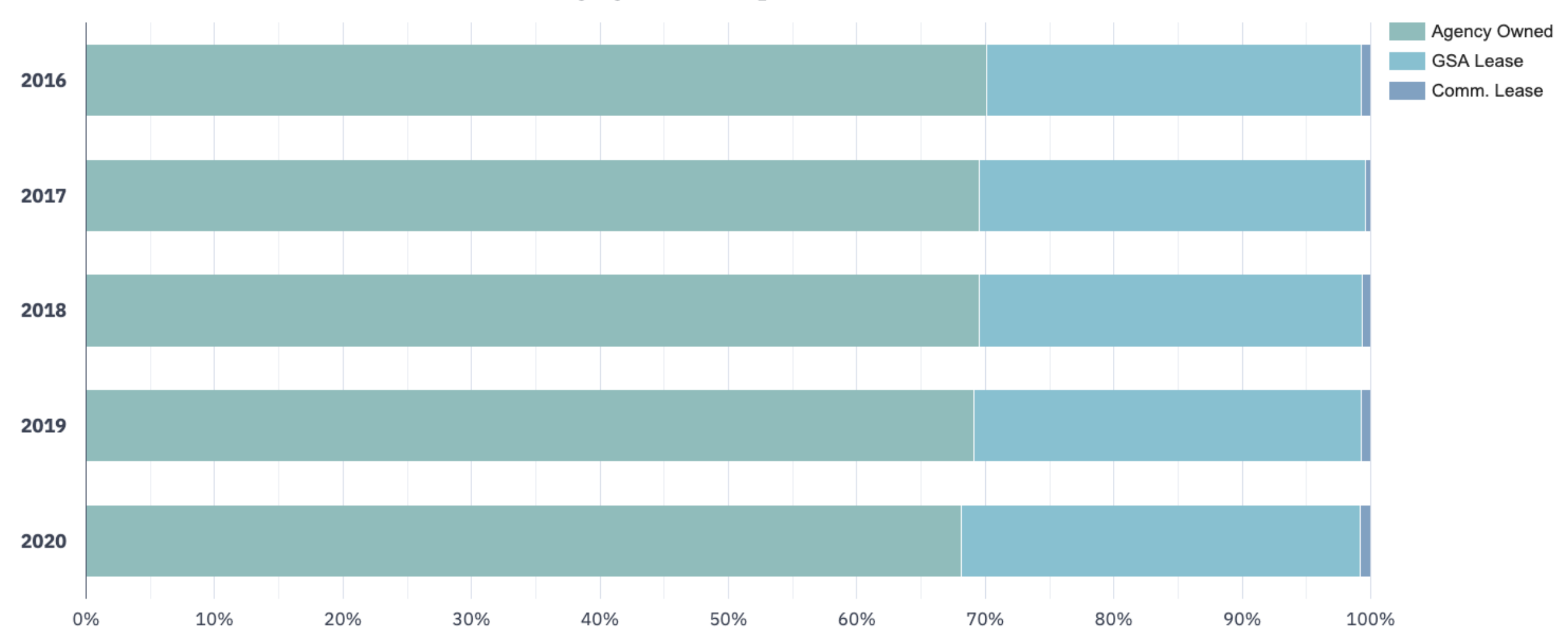




\section{FY 2020 Federal Fleet Dataset: 5-Year Trends}

Vehicle Inventory

FAST FY 2020 Dataset: Vehicle Inventory by Class

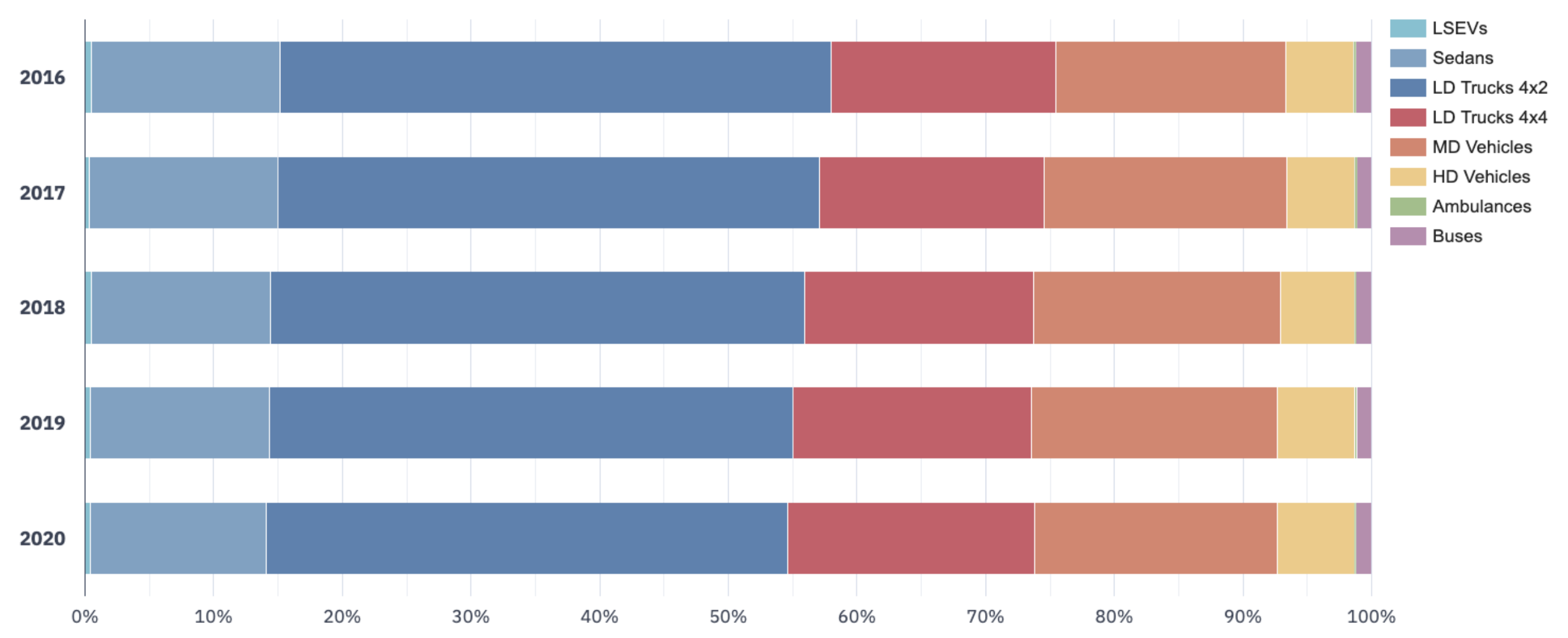




\section{FY 2020 Federal Fleet Dataset: 5-Year Trends}

Vehicle Inventory

FAST FY 2020 Dataset: Vehicle Inventory by Fuel Type \& Configuration

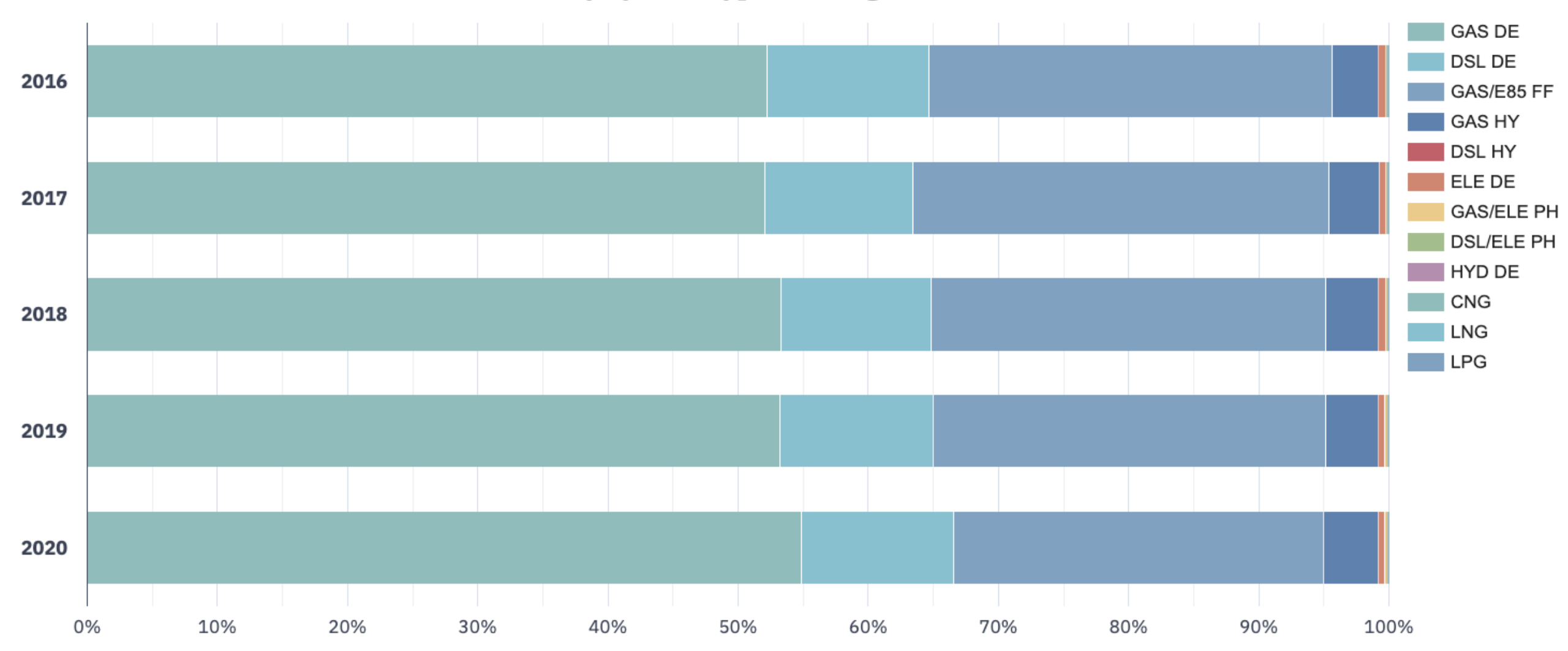




\section{FY 2020 Federal Fleet Dataset: 5-Year Trends}

Vehicle Inventory

FAST FY 2020 Dataset: Vehicle Inventory by Fuel Type \& Configuration

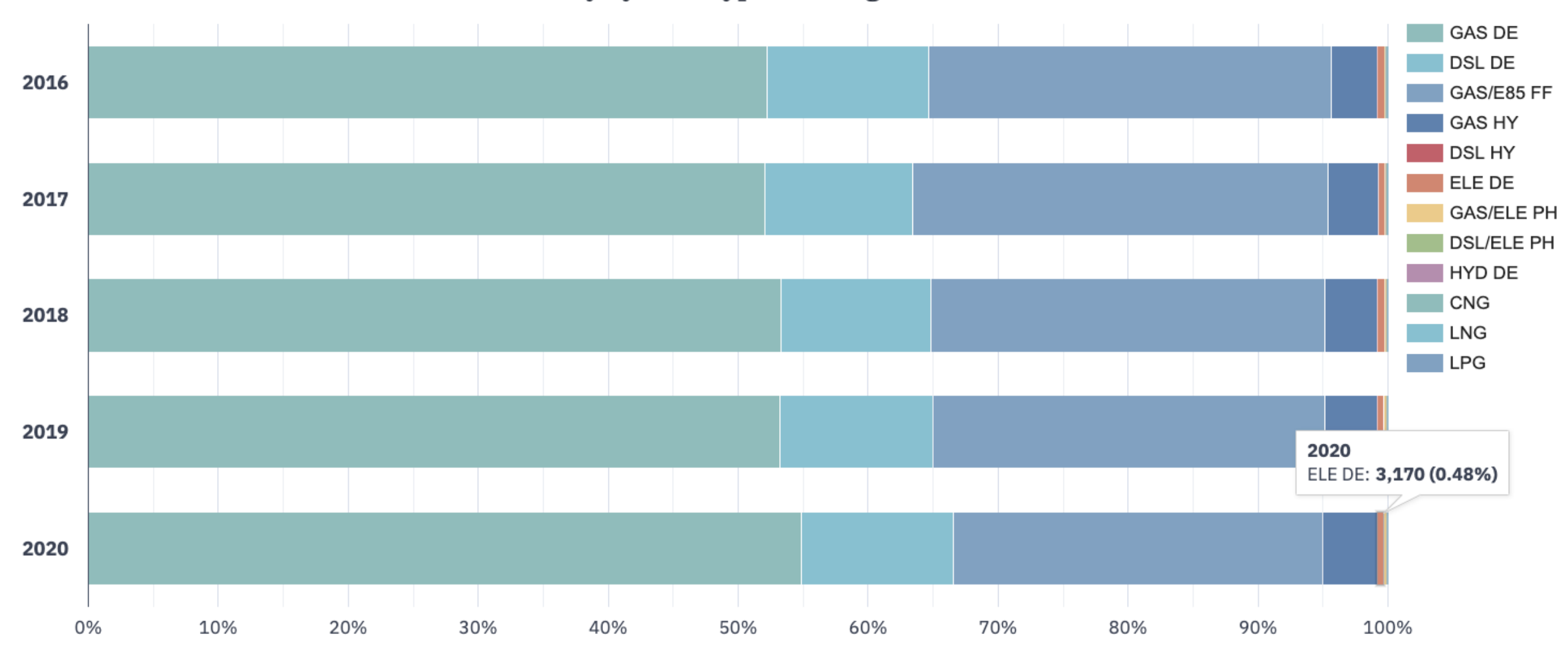




\section{FY 2020 Federal Fleet Dataset: 5-Year Trends}

Vehicle Acquisitions

FAST FY 2020 Dataset: Vehicle Acquisitions by Fuel Type \& Configuration

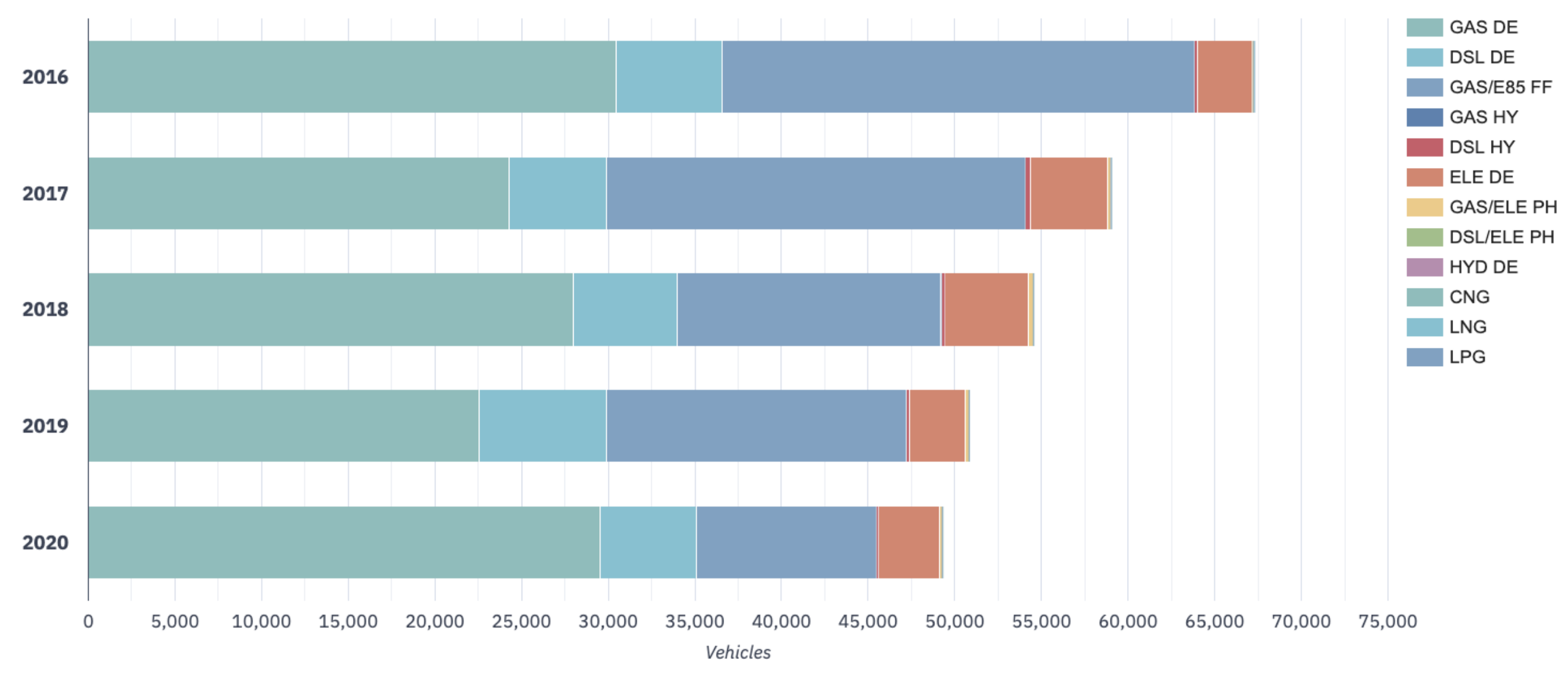




\section{FY 2020 Federal Federal Fleet Dataset: 5-Year Trends}

Vehicle Acquisitions

FAST FY 2020 Dataset: Vehicle Acquisitions by Fuel Type \& Configuration

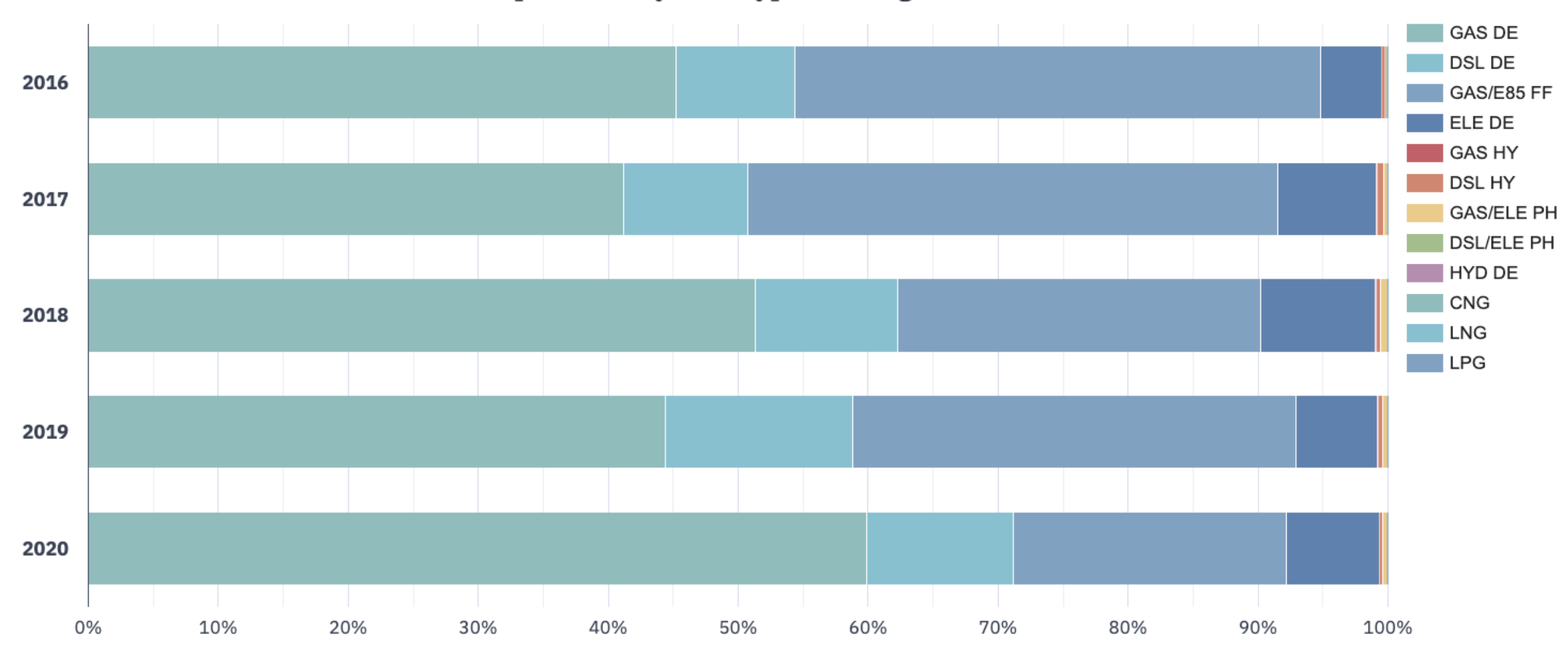




\section{FY 2020 Federal Fleet Dataset: 5-Year Trends}

Vehicle Acquisitions

FAST FY 2020 Dataset: Vehicle Acquisitions by Class

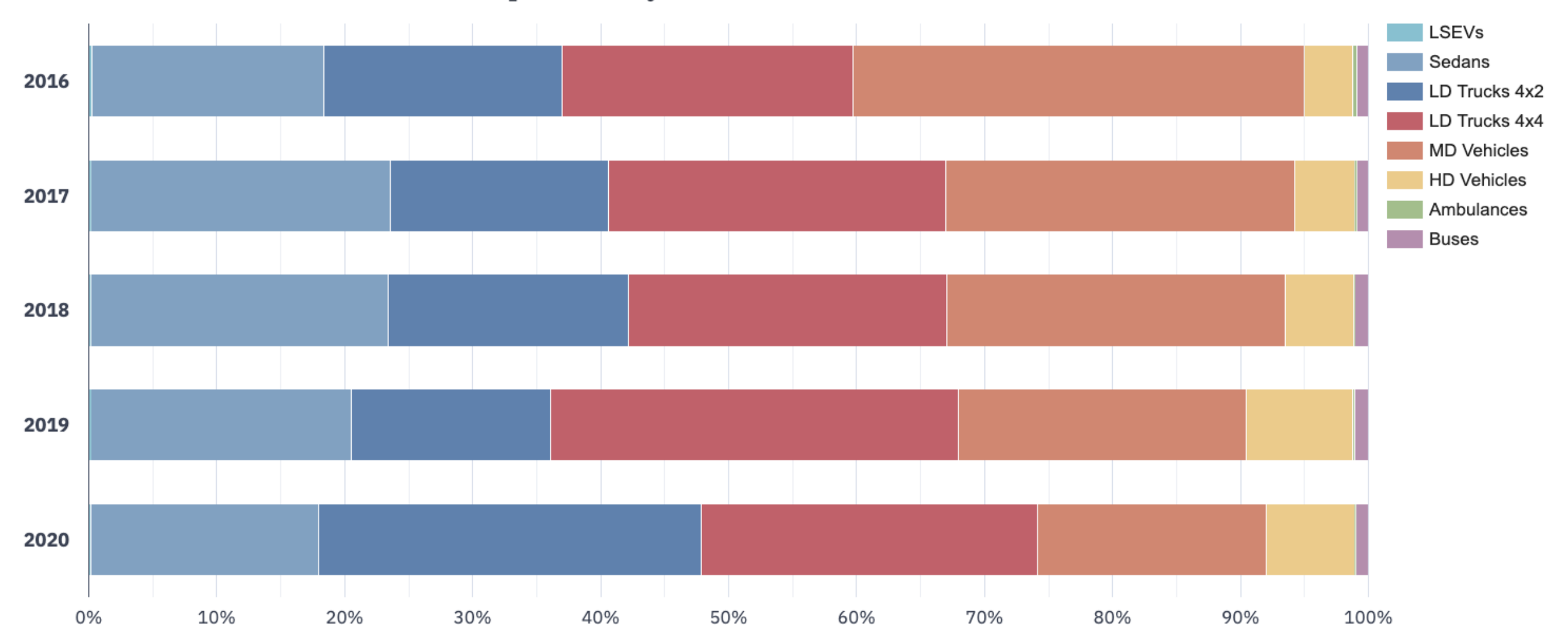




\section{FY 2020 Federal Fleet Dataset: 5-Year Trends}

Fuel Consumption

FAST FY 2020 Dataset: Fuel Consumption by Fuel Type

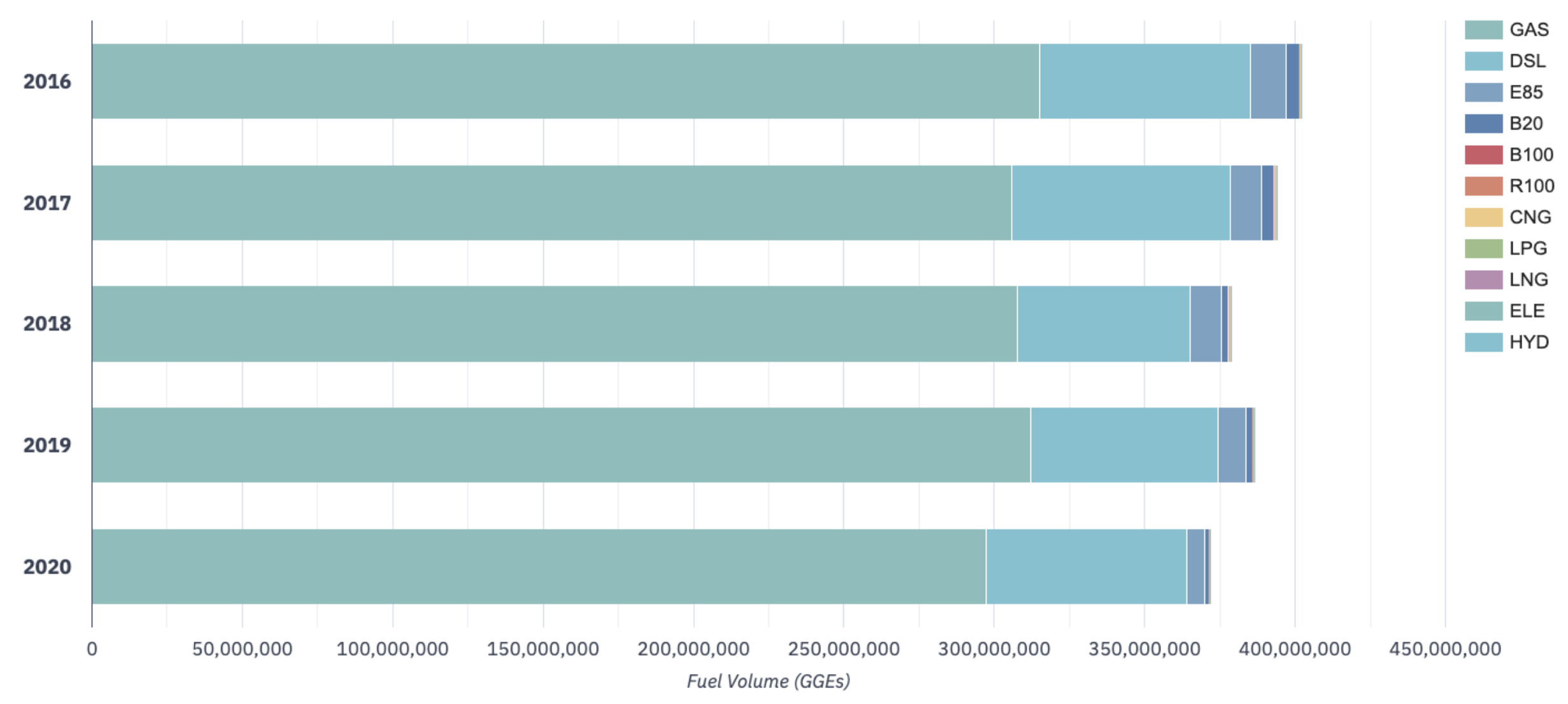




\section{FY 2020 Federal Fleet Dataset: 5-Year Trends}

Fuel Consumption

FAST FY 2020 Dataset: Fuel Consumption by Fuel Type

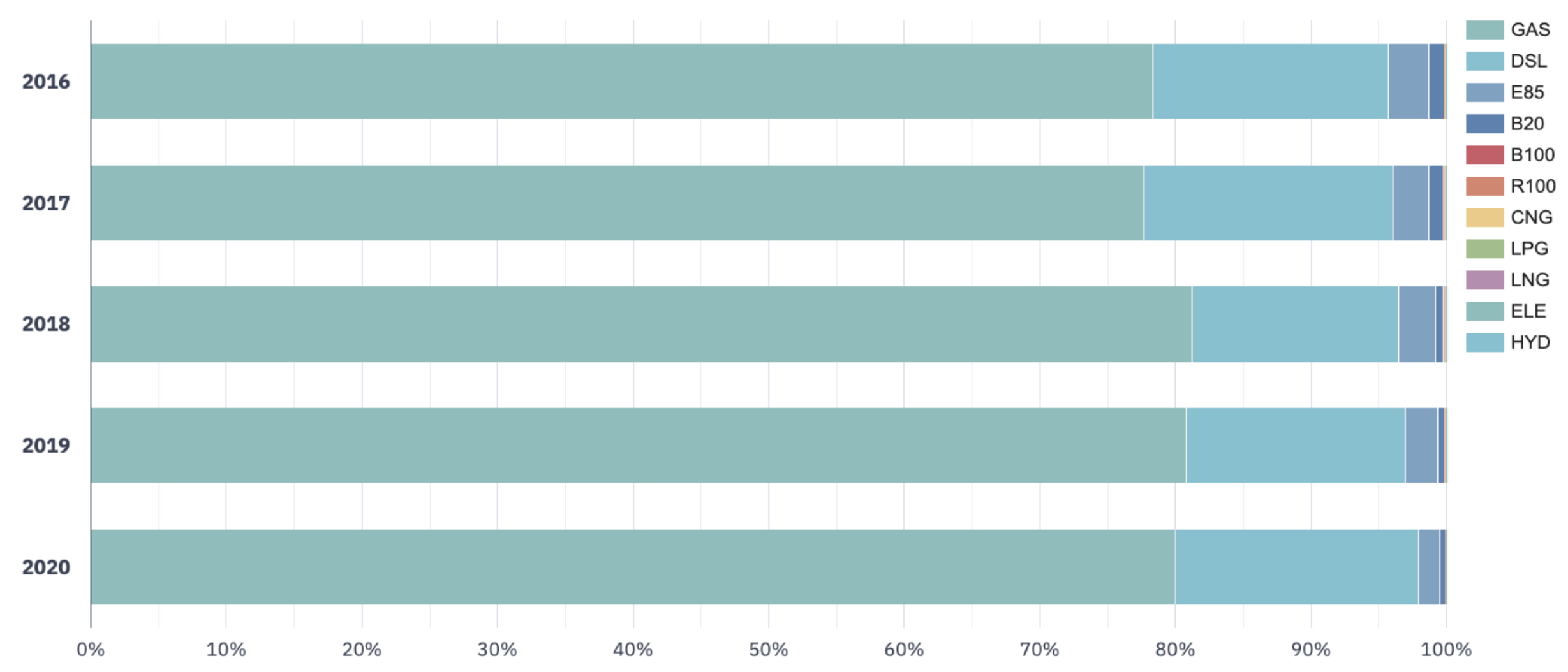




\section{FY 2020 Federal Fleet Dataset: 5-Year Trends}

\section{Operating Costs}

FAST FY 2020 Dataset: Fleet Costs by Cost Category

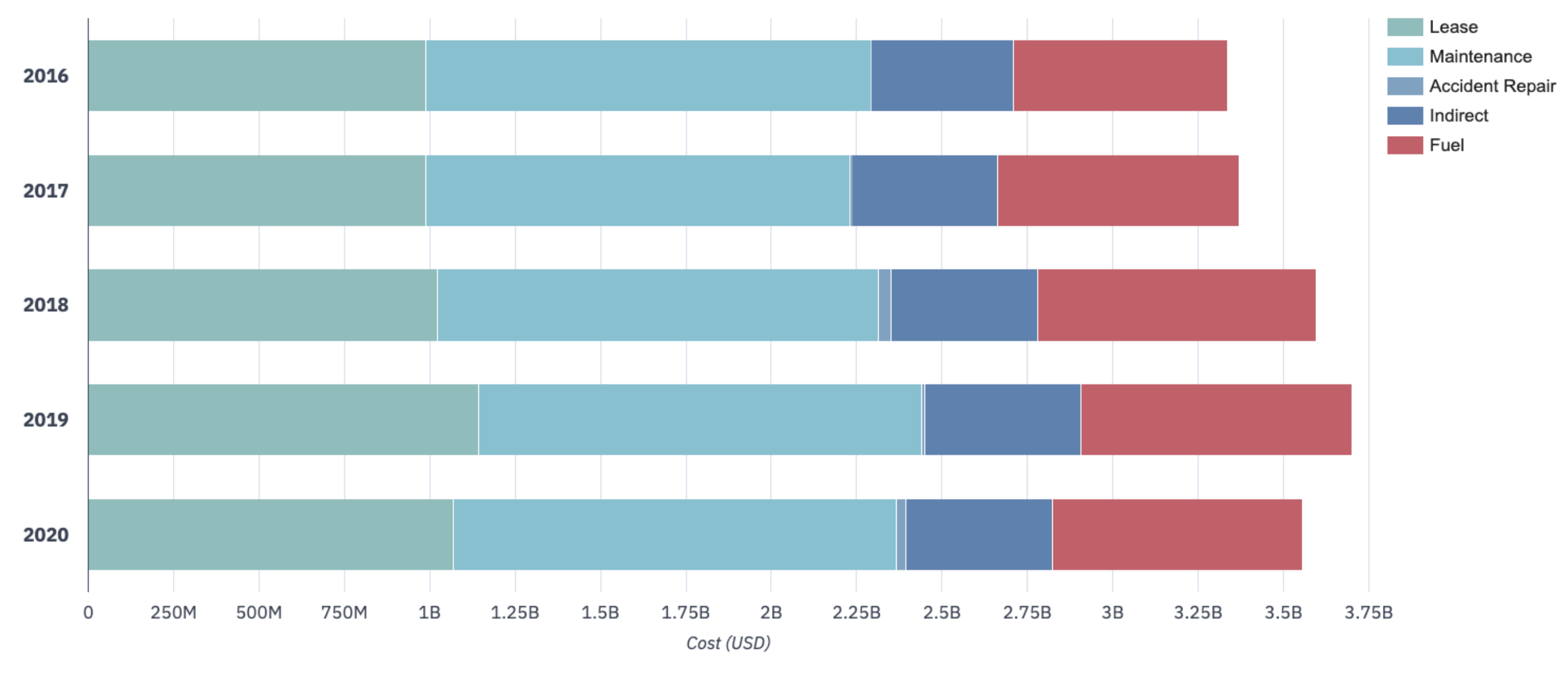




\section{FY 2020 Federal Fleet Dataset: 5-Year Trends}

\section{Operating Costs}

FAST FY 2020 Dataset: Fleet Costs by Cost Category

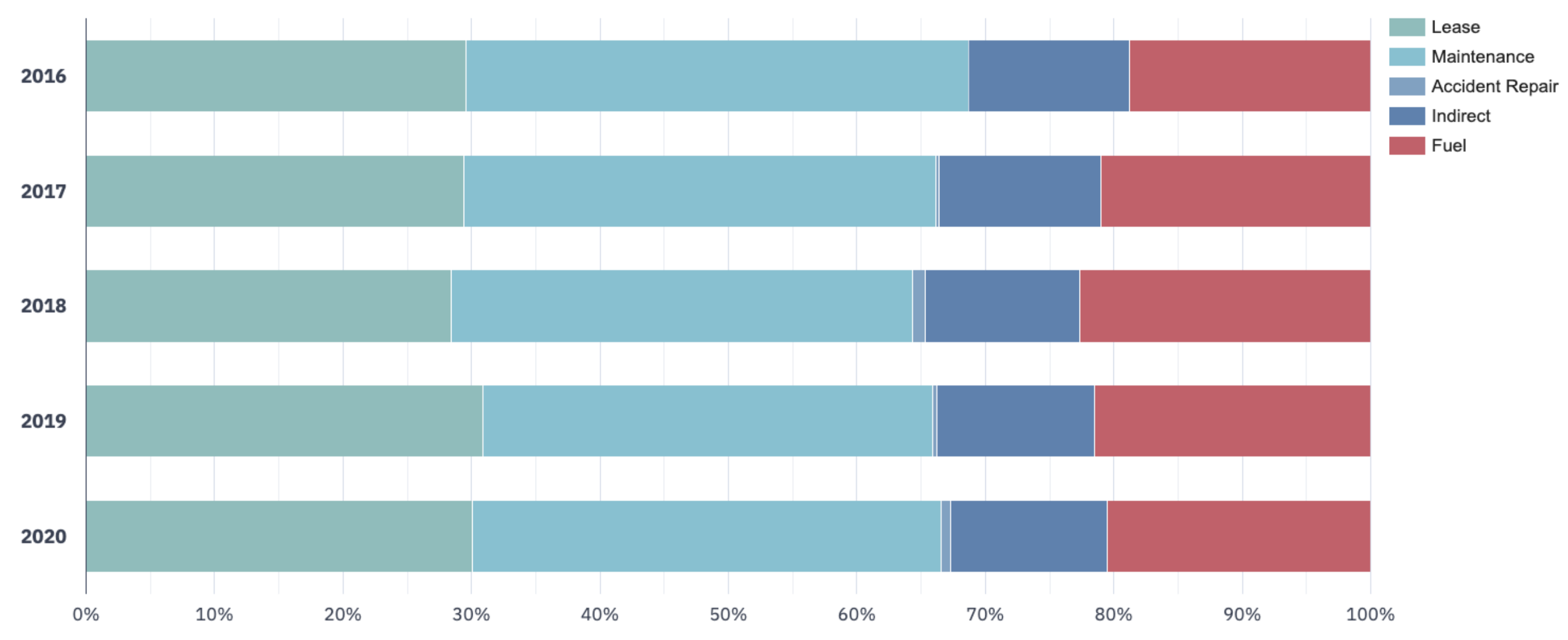




\section{FY 2020 Federal Fleet Dataset: Year-to-Year}

\section{Comparison}

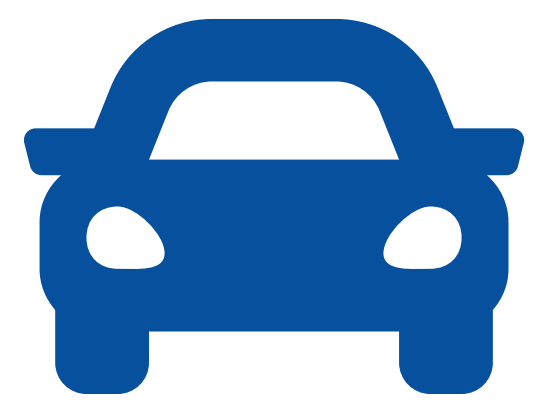

Inventory $658 \mathrm{~K}$

$+1.9 \%$

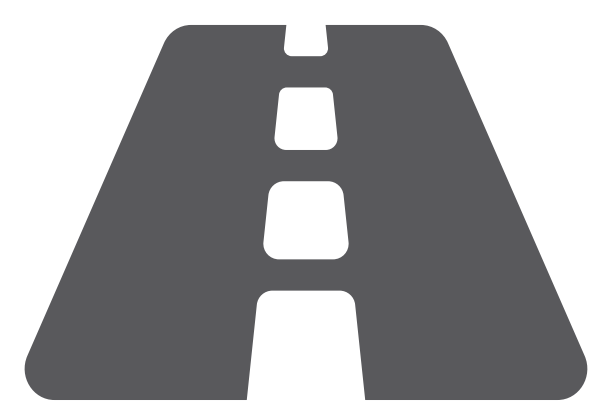

$\underline{\text { Miles }}$

4.16B

$-7.1 \%$

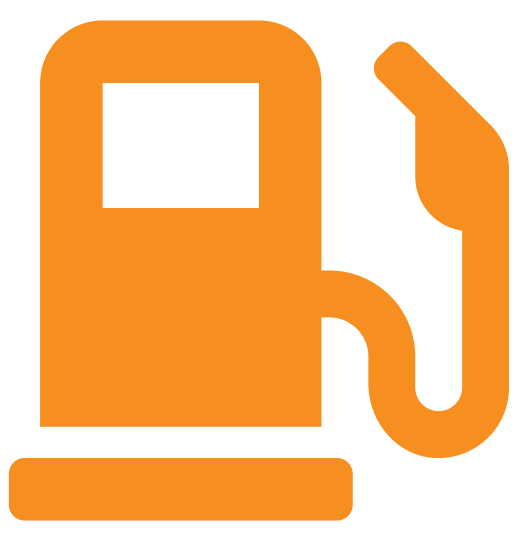

$\underline{\text { Fuel }}$

$372 \mathrm{M} \mathrm{GGE}$

$-3.8 \%$

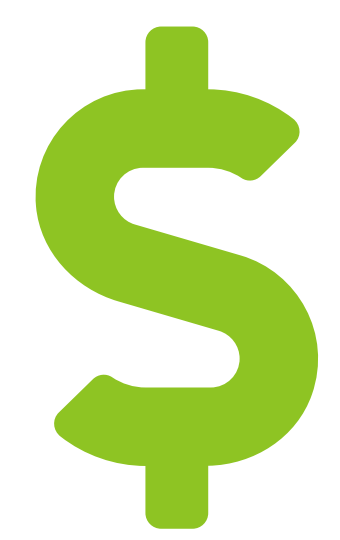

Cost $\$ 3.55 \mathrm{~B}$

$-3.9 \%$ 


\section{FY 2020 Federal Fleet Dataset: Year-to-Year}

\section{Comparison}

FAST FY 2020 Dataset: Agency Change in Fleet Miles (\%)

20

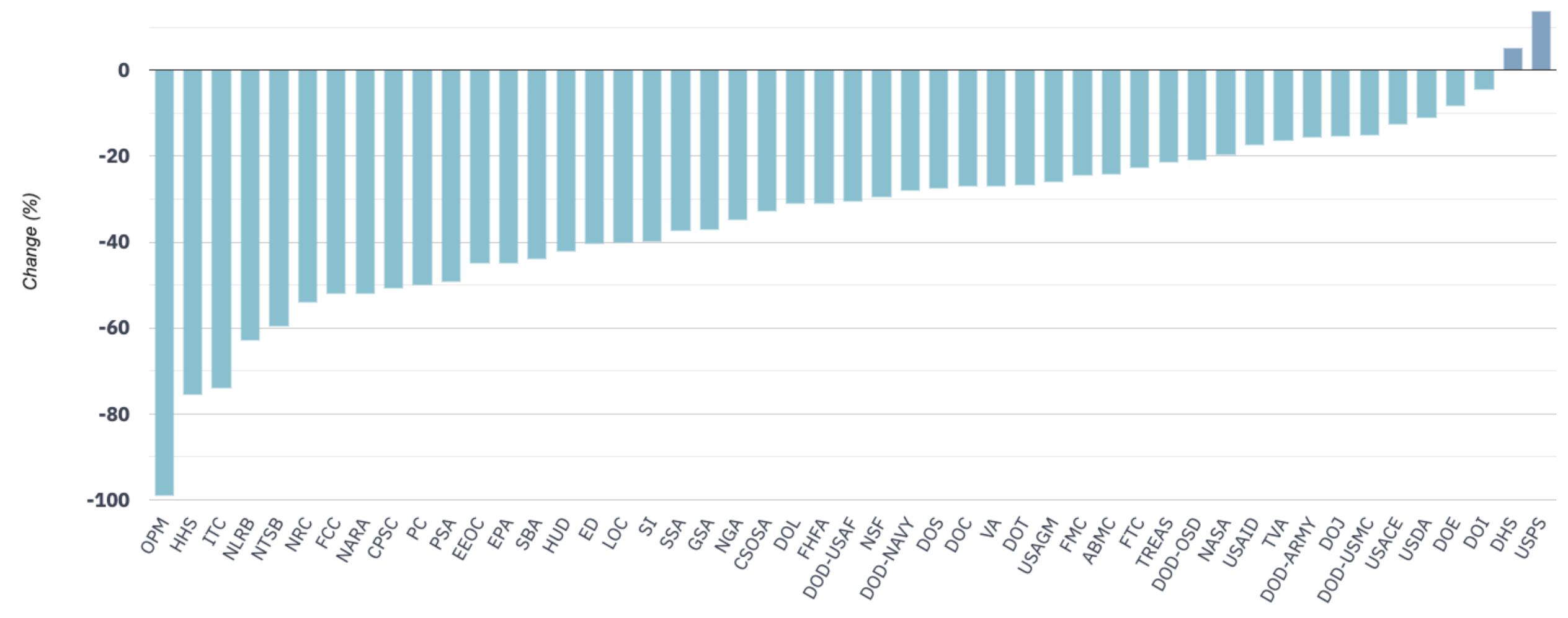




\section{FY 2020 Federal Fleet Dataset: Year-to-Year Comparison}

FAST FY 2020 Dataset: Agency Change in Fleet Miles

$200,000,000$

$150,000,000$

$100,000,000$

$50,000,000$

0

$-50,000,000$

$-100,000,000$

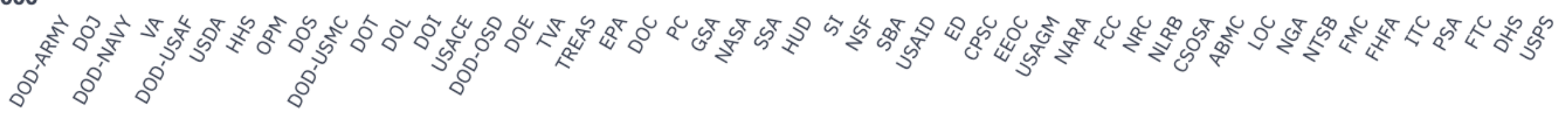




\section{FY 2020 Federal Fleet Dataset: Year-to-Year}

\section{Comparison}

FAST FY 2020 Dataset: Agency Change in Fleet Miles (\%)

20

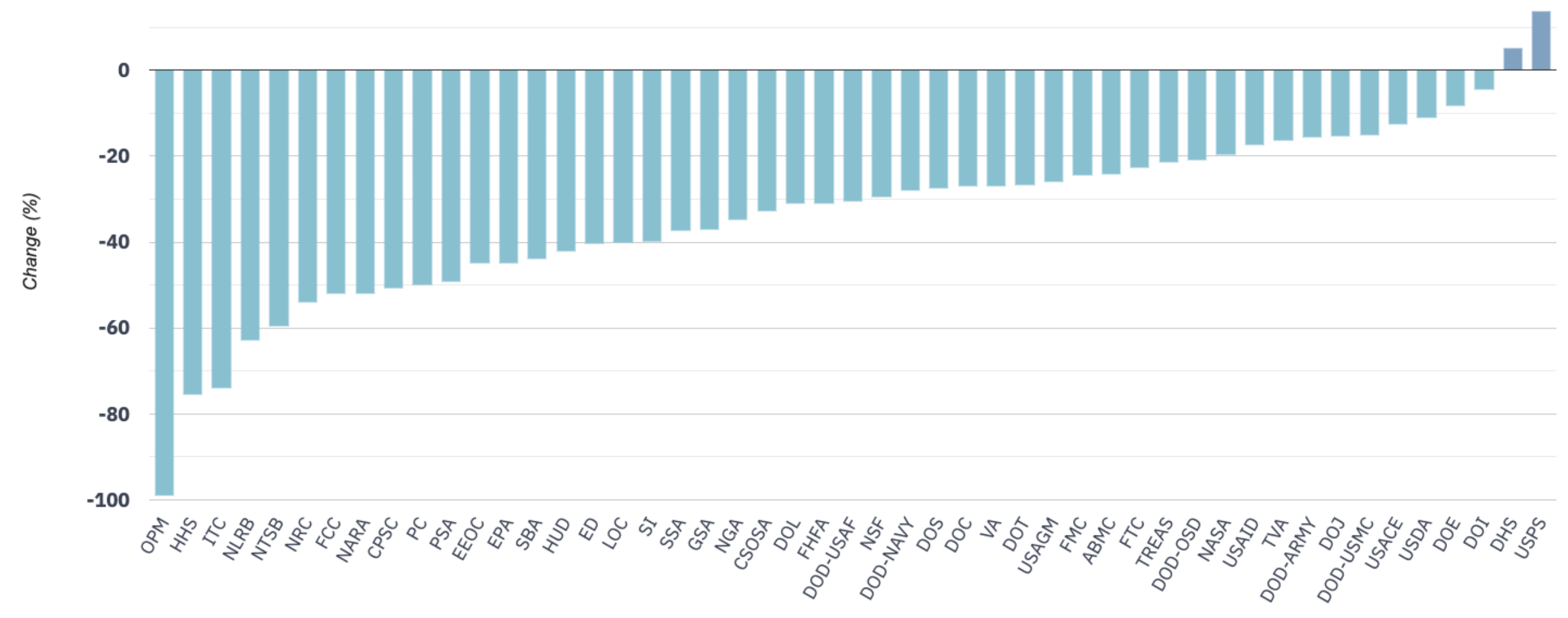




\section{FY 2020 Federal Fleet Dataset: Year-to-Year}

\section{Comparison}

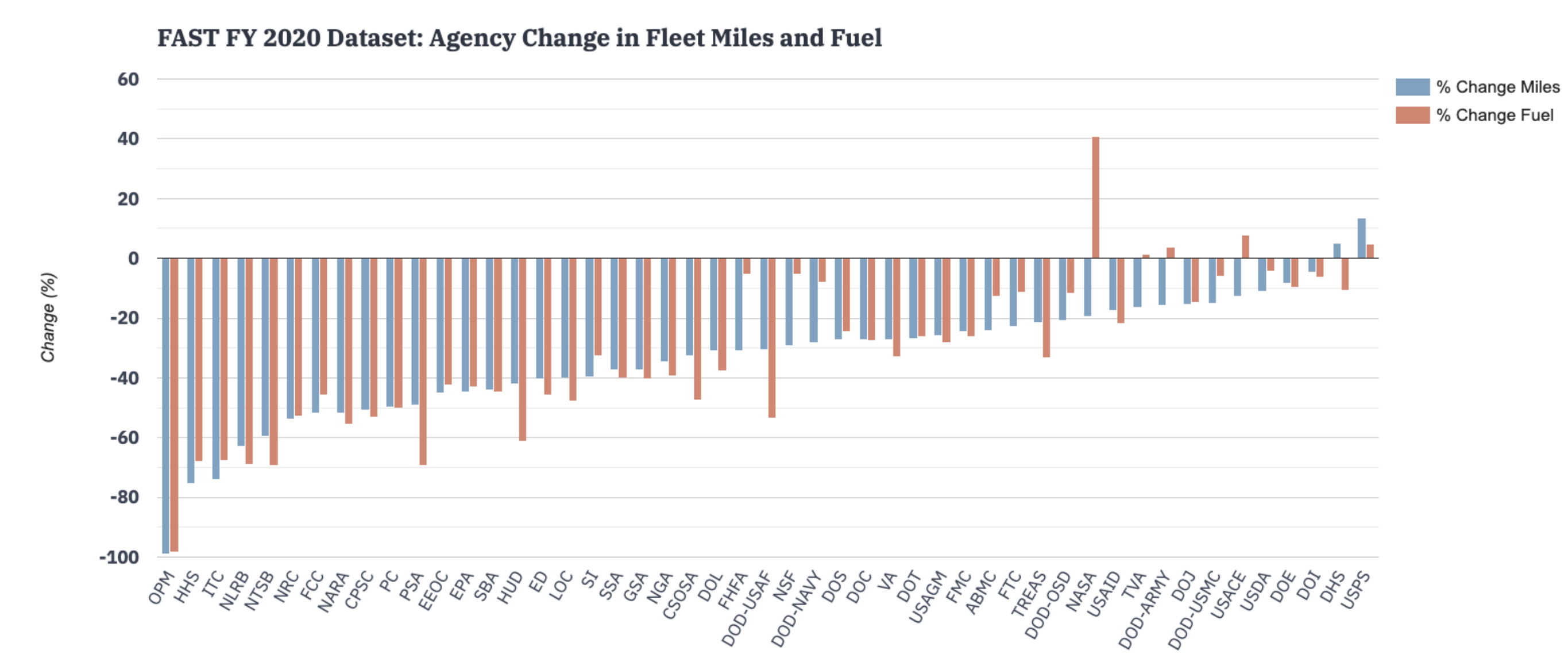




\section{FY 2020 Federal Fleet Dataset: Year-to-Year Comparison}

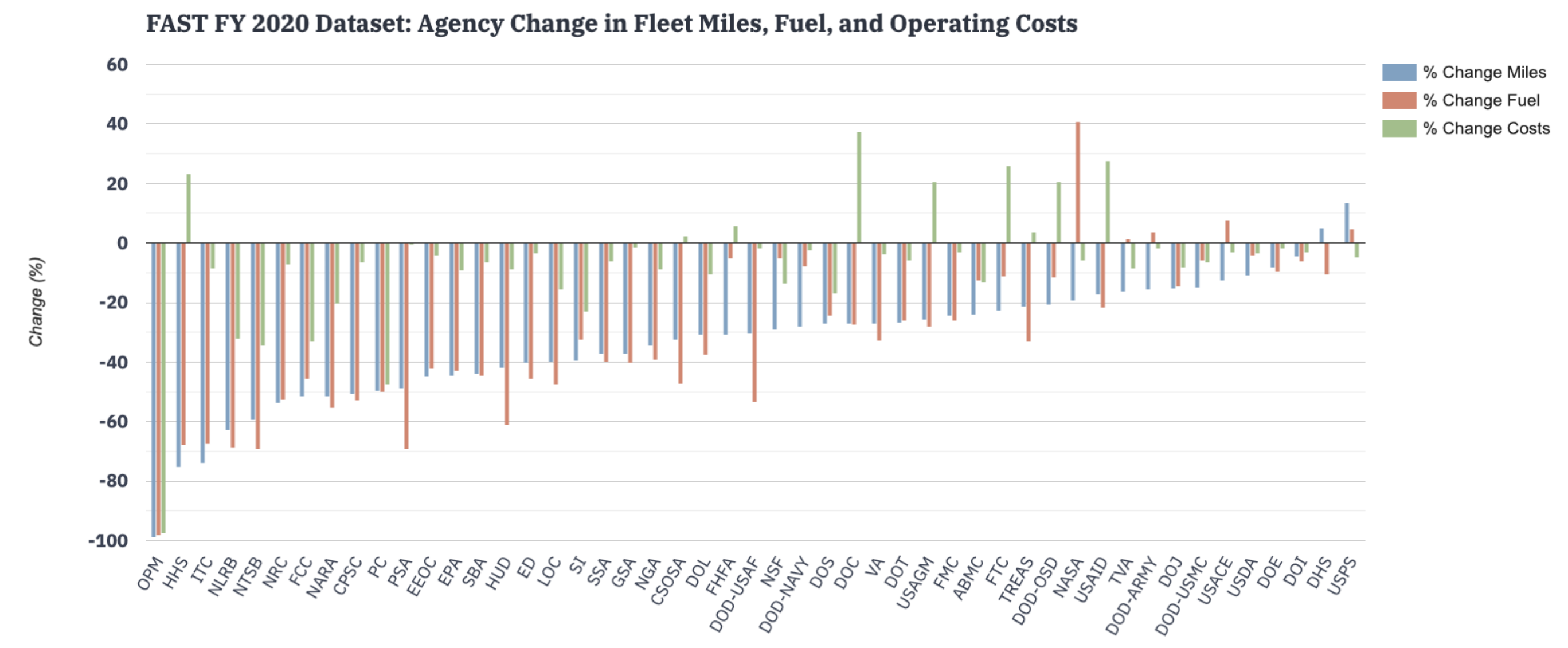




\section{FY 2020 Federal Fleet Dataset: Review and}

\section{Corrections}

Review Process and Results

- FAST management team reviewed agency submissions

- Written feedback provided to agencies (late January 2021)

- Agencies responded to any noted potential issues

- Agencies granted short period to

FAST FY 2020 Dataset: Dataset Review

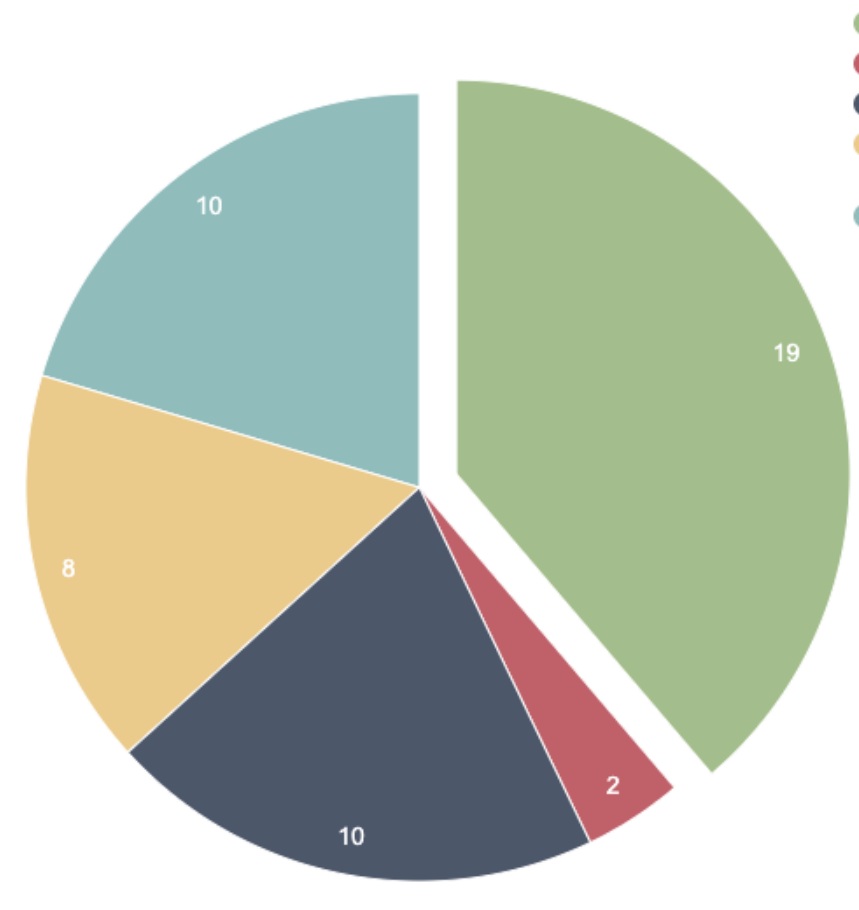

No significant issues noted

Issues: No response

Issues: No corrections

Corrections: No significant

improvement

Corrections: Improvement submit corrections where feasible (due late February 2021) 


\section{FY 2020 Federal Fleet Dataset: Review and}

\section{Corrections}

FAST FY 2020 Dataset: Dataset Review

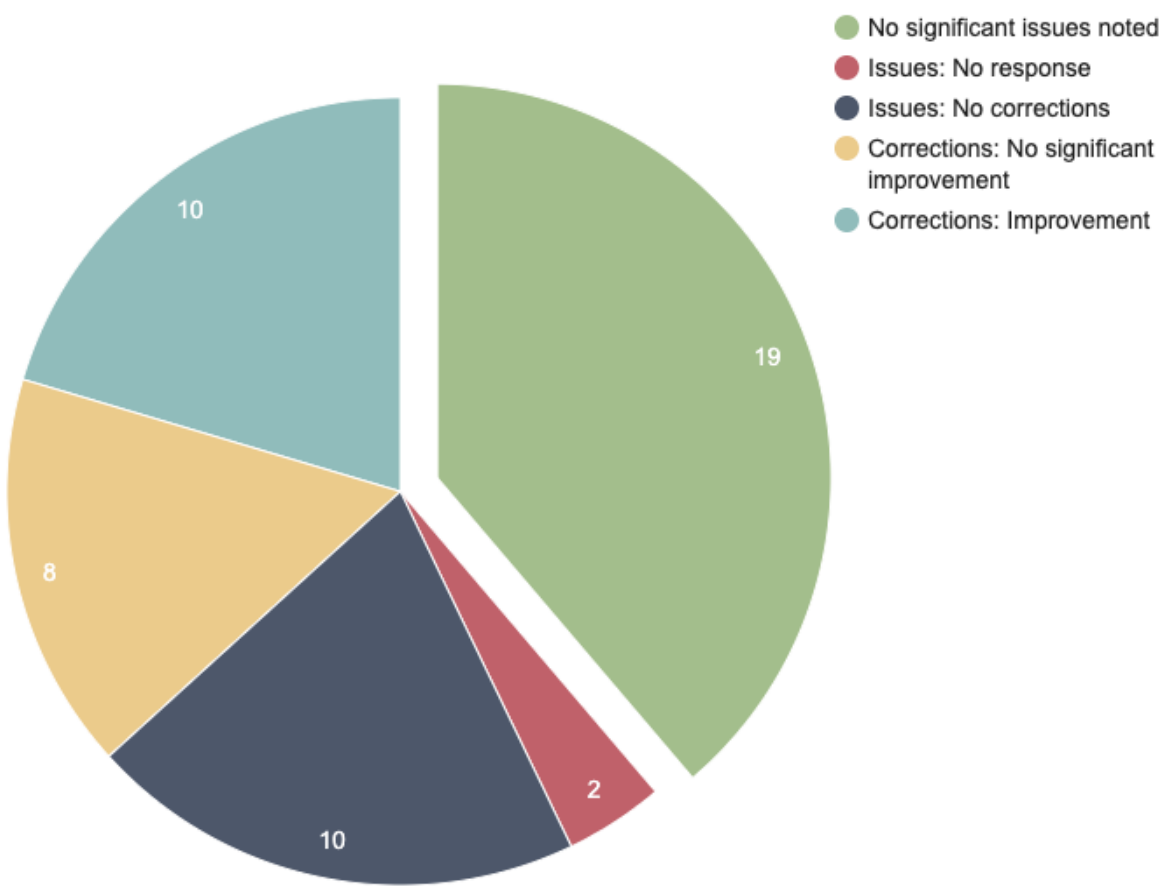

FAST FY 2019 Dataset: Dataset Review

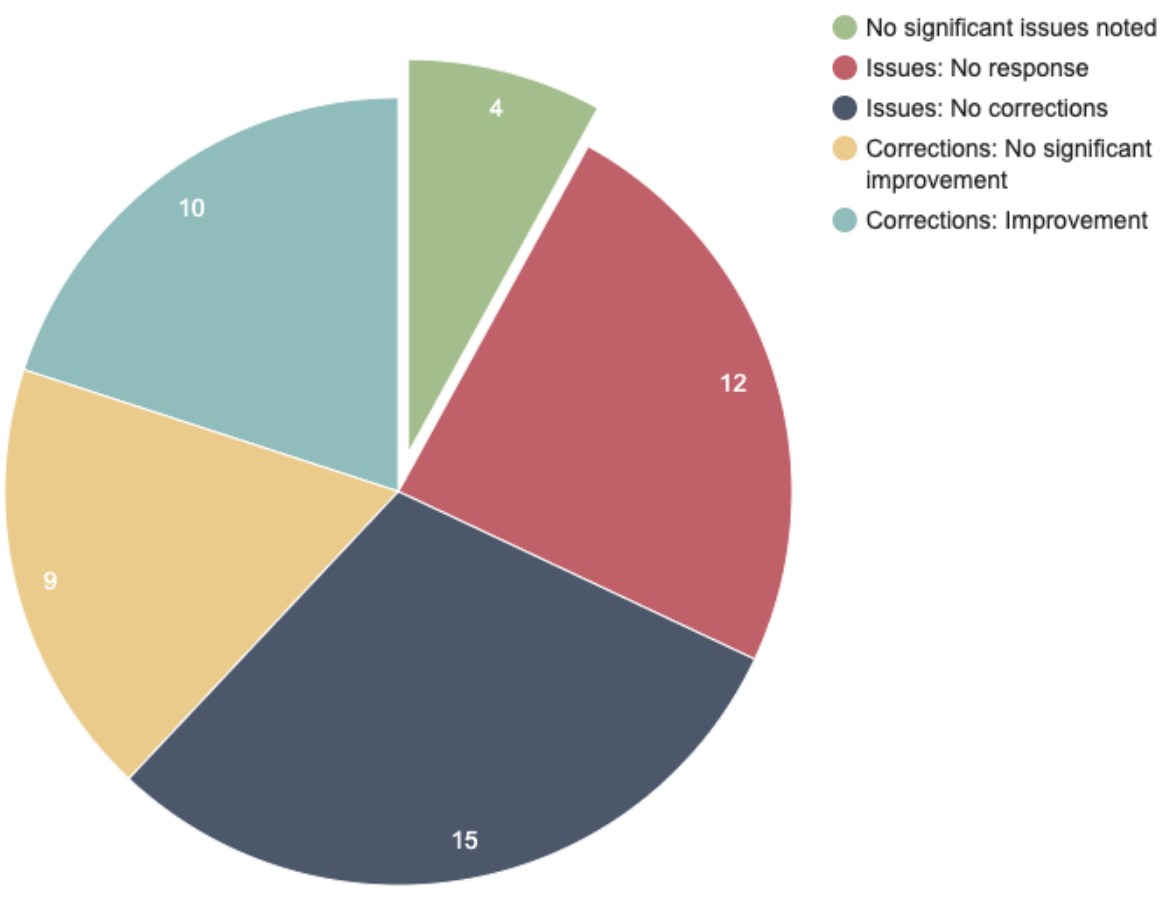




\section{FY 2020 Federal Fleet Dataset: Review and}

\section{Corrections}

Impact of Agency Corrections

- Review Measure 6: Invalid vehicle fuel efficiencies

- Agency corrections reduced issues by $19 \%$ (3,500 vehicles)

- 5 agencies addressed over $80 \%$ of the identified vehicles in their submissions

- Review Measure 7(d): Previously-reported vehicles missing

- 3 agencies reduced these by 15\%, 36\%, 100\% (adding almost 200 vehicles)

- Review Measure 8: Use of "placeholder" vehicle attributes

- 12 agencies reduced these:

- Over 1,400 fewer vehicles with placeholders

- Eliminated over 1,200 blocking placeholders, 700 flagging placeholders

- 2 agencies eliminated over $70 \%$ of their placeholders 


\section{FY 2020 Federal Fleet Dataset}

Important Activity After the Data Call

\section{- Fleet fuel cost data finalized}

- FAST's "Fleet Fuel Consumption Report for Greenhouse Gas Reporting" finalized

- Summaries for all agencies transmitted to DOE's Federal Energy Management

Program

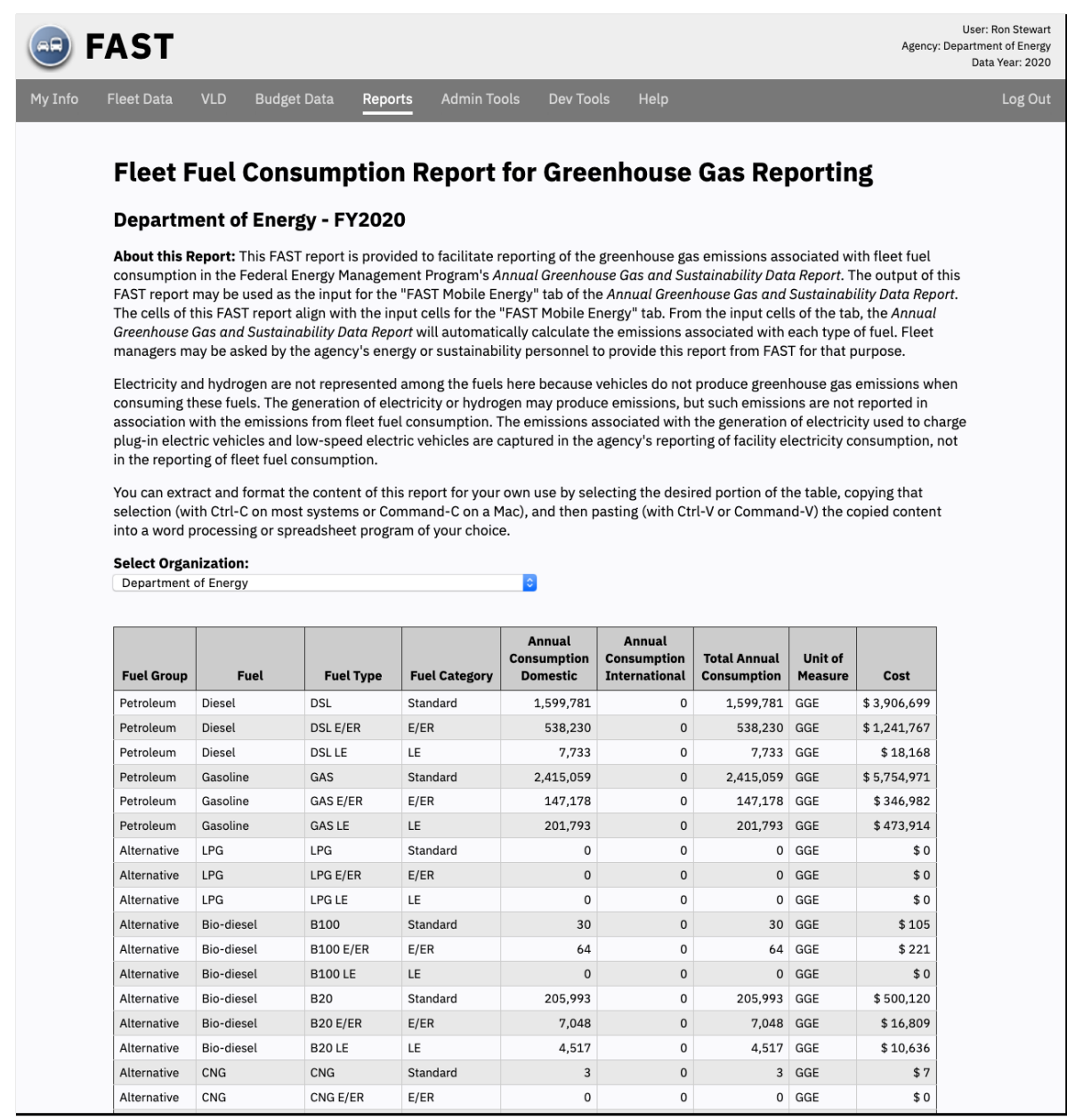




\section{FY 2020 Federal Fleet Dataset}

Important Activity After the Data Call

- EPAct AFV acquisition compliance analysis is complete

- Same process as FY 2019: vehicle location used to determine vehicle coverage / exemption status

- Now reflected on FAST's "EPAct AFV Acquisition Compliance Report"

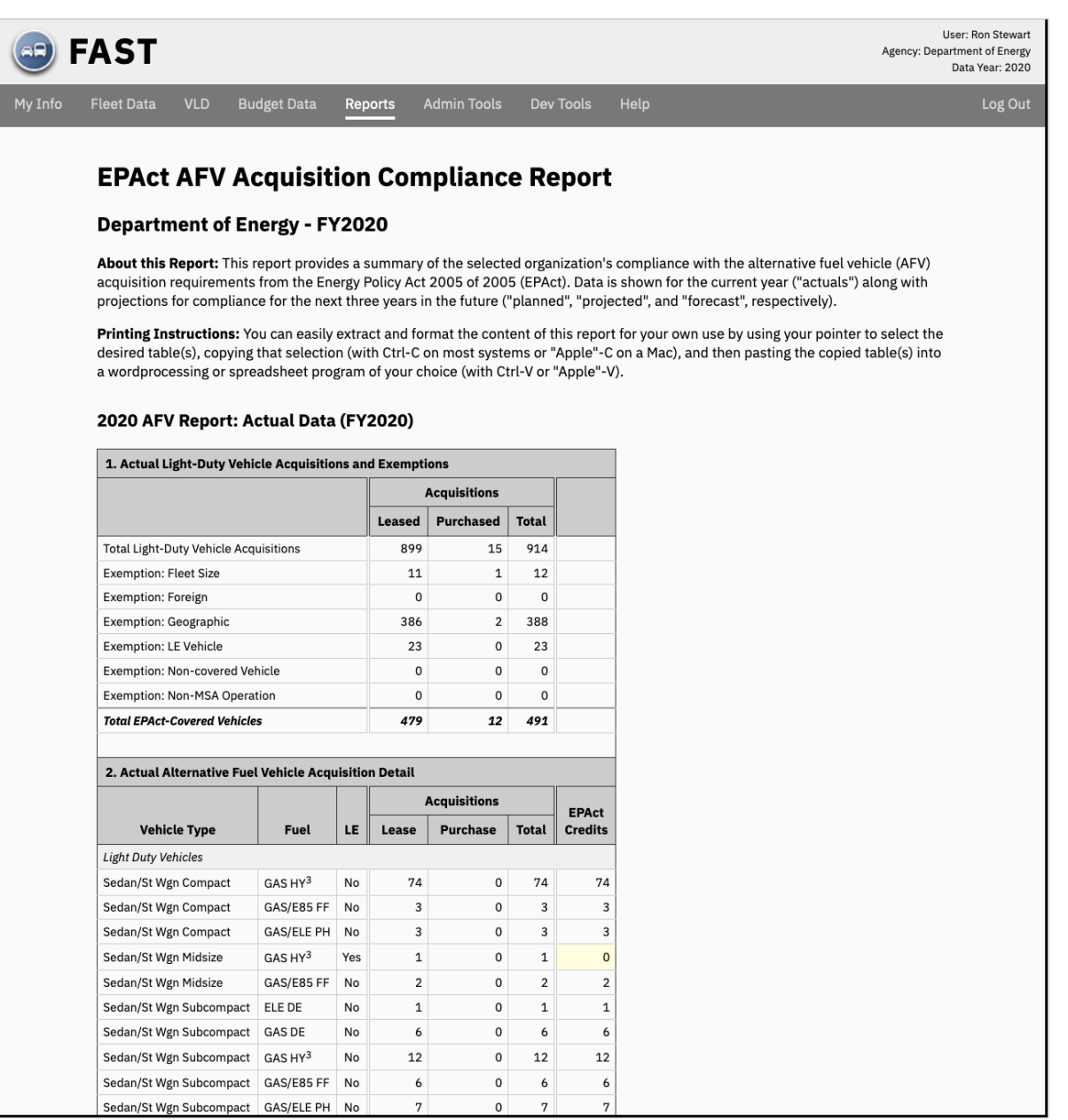




\section{FY 2020 Federal Fleet Dataset}

Important Activity After the Data Call

- Other Compliance and Reporting Activities

- Review and incorporation of fueling center and EVSE inventory submissions into DOE's Alternative Fuel Data Center

- EISA 2007 Section 141 compliance summary transmitted to EPA

- Preparation of OMB sustainability scorecards

- EPAct 2005 Section 701 compliance analysis

- Preparation of GSA's Federal Fleet Report data tables and related products

- Preparation for FY 2021 Reporting

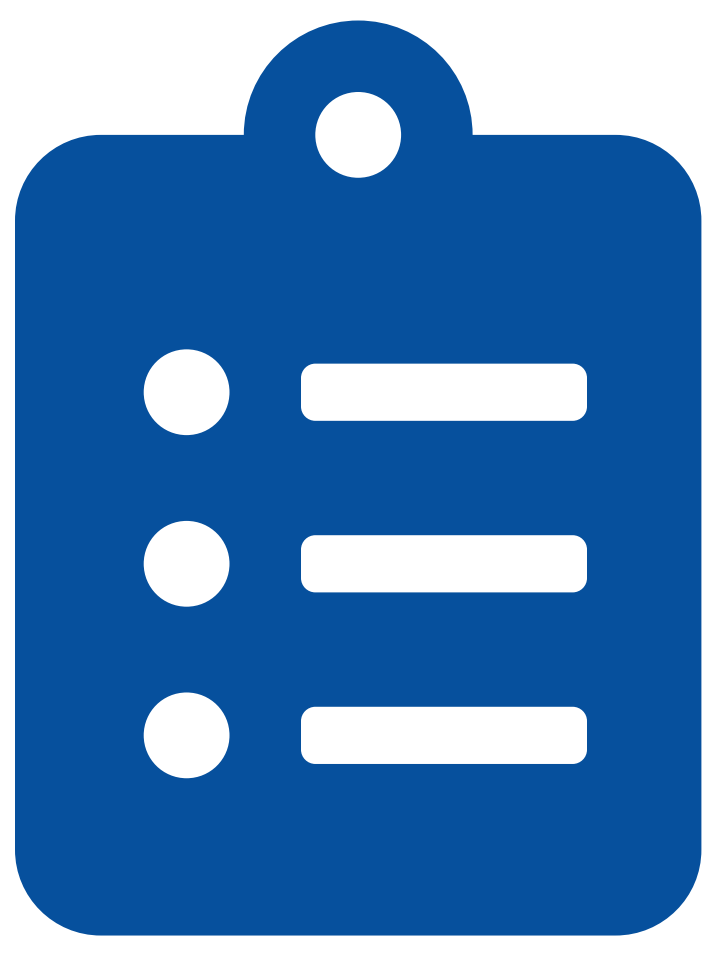




\section{FAST: FY 2020 Federal Fleet Dataset}

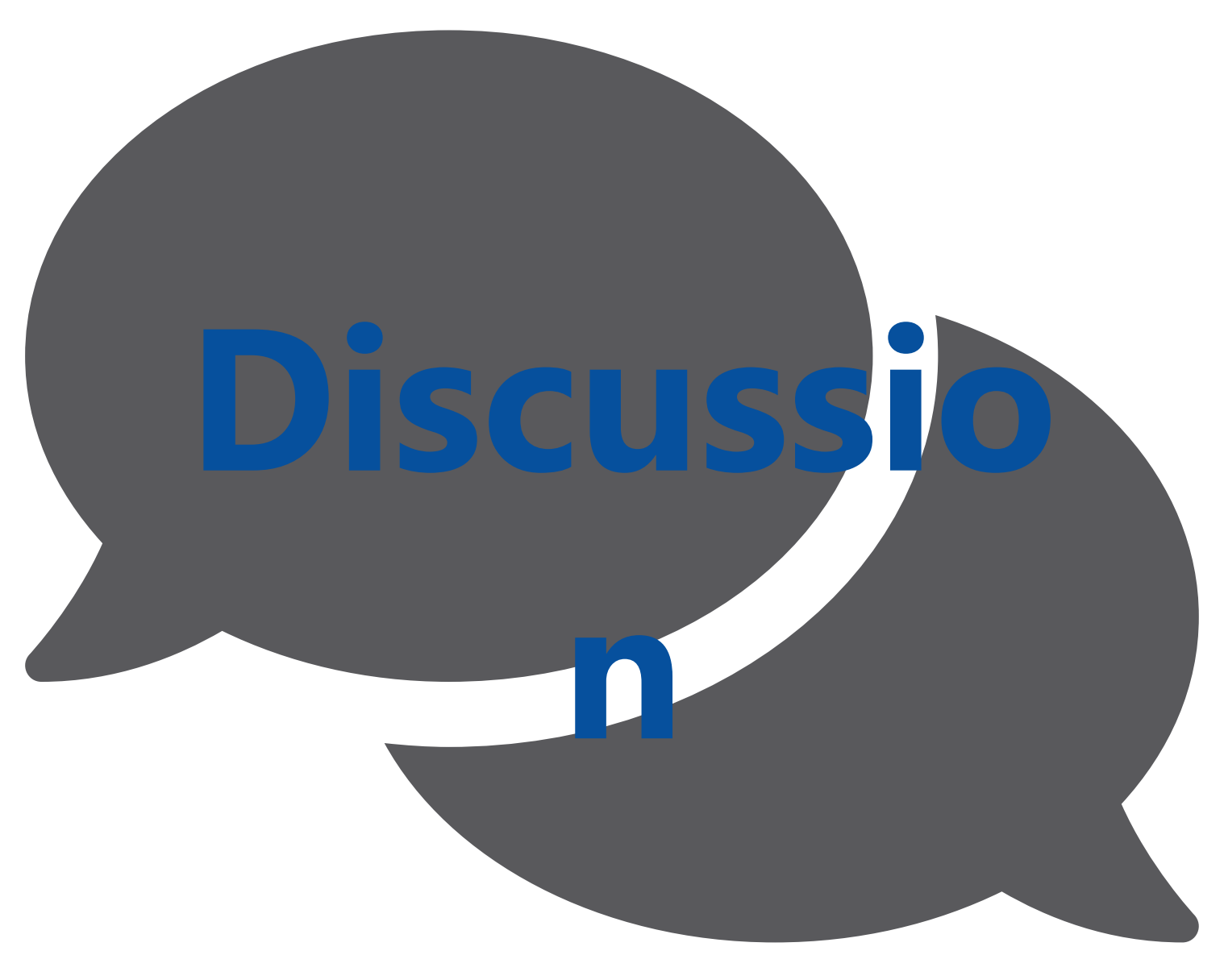




\section{FAST Program Points of Contact}

- DOE Federal Energy Management Program

- Lay Wrobel

Jay.Wrobel@ee.doe.gov

- Kndall Kam

Kendall.Kam@ee.doe.gov

- GSA Office of Government-wide Policy

- Surmes Vogelsinger

James.Vogelsinger@gsa.gov

- Patrick McConnell

Patrick.McConnell@gsa.gov
- FAST Support Team

- FASTsupport@inl.gov

- $@$ @FASTdevs

- FAST

- https://fastweb.inl.gov/

- FASగुHelp

- https://fastweb.inl.gov/help/ 


\title{
Ron Stewart
}

\author{
FAST Team Lead
}

Idaho National Laboratory

ron.stewart@inl.gov 